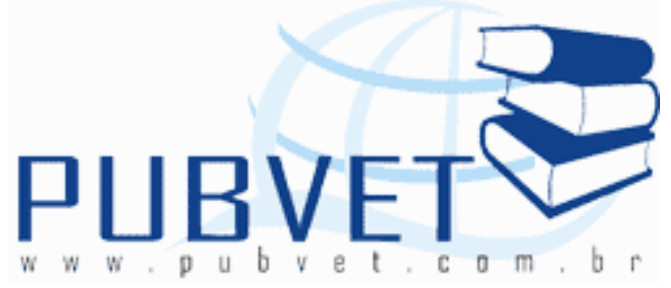

PUBVET, Publicações em Medicina Veterinária e Zootecnia.

\title{
Comportamento da produção de leite de cabras Saanen e mestiças Boer-Saanen
}

\footnotetext{
Laís Aberrachid Jacopini $^{1 *}$, Daniela Andressa Lino Lourenço ${ }^{3}$, Carlos Antônio Lopes de Oliveira ${ }^{4}$, Carlos Alberto dos Santos Deroide ${ }^{2}$, Elias Nunes Martins ${ }^{4}$
}

${ }^{1}$ Parte da monografia apresentada pelo autor ao Curso de Zootecnia da Universidade Estadual de Maringá, para obtenção do grau de Bacharel em Zootecnia

2 Aluno de graduação do curso de Zootecnia da Universidade Estadual de Maringá, Maringá, Paraná

${ }^{3}$ Aluno de pós-graduação do curso de Zootecnia da Universidade Estadual de Maringá, Maringá, Paraná

4 Professores do Departamento de Zootecnia da Universidade Estadual de Maringá, Maringá, Paraná, Av. Colombo, 5790, 87020-900, Maringá, Paraná, Brasil.*E-mail: lais-jacopini@hotmail.com

\section{Resumo}

O presente trabalho teve por objetivo analisar o comportamento da produção de leite de cabras Saanen e mestiças Boer-Saanen através dos parâmetros da curva de lactação. Foram utilizadas oito cabras Saanen e nove cabras 3/4 Boer$1 / 4$ Saanen para comparar o comportamento de suas produções. Para tal comparação foi utilizado o modelo não linear de Wood por meio de análise bayesiana para determinação dos parâmetros da curva de lactação, sendo 
eles: A - produção inicial de leite; B- taxa de acréscimo de produção até o pico e C- taxa de declínio de produção após o pico. Os valores dos parâmetros A e B foram maiores para as cabras da raça Saanen, mostrando que estes animais apresentam maior produção de leite inicial e taxa de acréscimo até o pico, respectivamente. Observou-se que tanto as cabras puras quanto as mestiças apresentaram taxa de declínio de produção semelhante, já que não se verificou diferença para o parâmetro C. Cabras Saanen apresentam maior produção de leite em todas as fases da curva, consequentemente, maior persistência e produção de leite.

\title{
Trends of milk production in Saanen and crossbred Boer-Saanen goats
}

\begin{abstract}
The purpose of this study was to analyze the behavior of the milk production of Saanen goats and crossbred Boer-Saanen goats through the parameters of lactation curve. The behavior of the production was compared in eight Saanen goats and nine crossbred $3 / 4$ Boer-1/4 Saanen goats. For this comparison it was used the Wood nonlinear model in Bayesian analyses to acess the parameters of the lactation curve, namely: A the initial milk yield; $B$ - rate increase production to peak and $\mathrm{C}$-rate of production decline after the peak. The values of the parameters A and B were higher for Saanen goats, indicating that these animals have higher initial milk yield and rate of increase to peak, respectively. Both pure goats and crossbred goats showed similar rate of production decline, since no difference was found for the parameter $\mathrm{C}$.
\end{abstract}

\section{INTRODUÇÃO}

O desempenho da produção de leite de um animal durante a sua lactação pode ser visualizado através da curva de lactação, que é definida como a produção de leite por determinado período de tempo. A curva de lactação apresenta três fases que explicam a produção de leite do animal: uma 
fase inicial, logo após o parto, a taxa de acréscimo da produção até o pico e, finalmente, a taxa de declínio após o pico de produção.

Uma vez que produção de leite é uma característica que sofre influências de fatores como ordem da lactação, raça e idade da fêmea, alimentação, além da própria genética do animal. (RODRIGUES et al., 2006; MORAND-FEHR, 2005), ela se comporta de maneira diferente para cada animal, sendo importante saber qual o desempenho de cada um deles.

A comparação da forma da curva de lactação entre grupos de animais com diferentes composições raciais, idades ao parto e outros tratamentos de interesse, é importante, pois esta comparação permite conhecer a produção de leite de cada grupo, proporcionando um melhor controle da produção (GROENEWALD; VILJOEN, 2003). Além disso, a comparação da curva de lactação permitiria agrupar animais que tivessem a produção mais semelhante entre si, facilitando o manejo alimentar, reprodutivo e também de descarte e seleção, reduzindo os custos de produção da atividade (VAL-ARREOLA et al., 2004; VANRADEN et al., 2006; CUNHA et al., 2010; GLÓRIA et al., 2010).

A importância da curva de lactação reside na caracterização ampla da produção do animal durante toda a lactação, podendo ser identificado: o tempo de ascensão ao pico, o pico de produção, o tempo de queda ou persistência da lactação, duração da lactação, duração do período seco, duração da gestação, além de quedas bruscas de produção, resposta a dietas e manejo, dentre outros fatores (REKAYA et al., 2000).

Segundo Oliveira et al. (2007), do ponto de vista genético, as curvas são importantes para o estabelecimento de estratégias capazes de otimizar a seleção e a busca de genótipos mais eficientes e rentáveis para o produtor.

Modelos de curva de lactação têm sido propostos e alguns foram testados em cabras. Na maioria dos estudos de curva de lactação tem-se adotado o modelo de Wood, pois permite a estimativa de características básicas da curva, como produção máxima de leite, tempo para se atingir essa produção e persistência, com apenas três parâmetros (RIBEIRO; PIMENTA FILHO, 1999). 
Em estudo com cabras mestiças Saanen, Macedo et al. (2001) concluíram que $o$ modelo de Wood não linear foi que o melhor descreveu o comportamento da curva de lactação, pois apresentou menor variância que os demais modelos testados.

Com o intuito de se obter estimativas cada vez mais precisas, mesmo quando o conjunto de dados é pequeno, a abordagem Bayesiana vem sendo cada vez mais utilizada em estudos envolvendo produção animal, incluindo curvas de lactação. Ela é baseada nos métodos de Monte Carlo em Cadeias de Markov, que consistem em processos iterativos para geração de amostras (GELMAN et al., 1997; CARDOSO, 2009; FARIA et al., 2007).

Objetivou-se analisar o comportamento da produção de leite de cabras Saanen e mestiças Boer - Saanen, por meio de comparação das curvas de lactação das mesmas, utilizando-se o modelo não linear de Wood ajustado pela abordagem Bayesiana.

\section{MATERIAL E MÉTODOS}

O trabalho foi conduzido no setor de Caprinocultura da Fazenda Experimental de Iguatemi - FEI, pertencente à Universidade Estadual de Maringá, no período de Agosto de 2009 a Julho de 2010. Foram utilizadas 17 cabras, sendo oito da raça Saanen e nove mestiças $3 / 4$ Boer- $1 / 4$ Saanen. Os animais foram ordenhados duas vezes por dia e tinham sua produção leiteira anotada individual e diariamente, para o controle leiteiro do rebanho.

As rações experimentais foram constituídas por farelo de soja, milho moído, mistura mineral e a silagem de milho, numa proporção volumoso: concentrado de 40:60. As composições percentuais e químico-bromatológicas da dieta podem ser visualizadas na Tabela 1. 
Tabela 1. Composições percentuais e químico-bromatológicas da dieta

\begin{tabular}{|c|c|}
\hline Alimentos & Nível \\
\hline Silagem de milho (\%) & 40,00 \\
\hline Milho moído (\%) & 42,53 \\
\hline Farelo de soja (\%) & 16,60 \\
\hline Calcário (\%) & 0,37 \\
\hline Mistura mineral $^{(1)}(\%)$ & 0,50 \\
\hline \multicolumn{2}{|l|}{ Nutrientes } \\
\hline Matéria Seca $(\%)$ & 65,45 \\
\hline Proteína Bruta (\%MS) & 16,08 \\
\hline Extrato Etéreo (\%MS) & 3,21 \\
\hline NDT (\%MS) & 75,43 \\
\hline
\end{tabular}

Para análise do comportamento da produção leiteira utilizou-se o modelo não linear de Wood (WOOD, 1967), lançando mão da metodologia Bayesiana para estimação dos parâmetros do modelo:

$$
Y=A n^{B} * \exp (-C n),
$$

Em que: $Y=$ produção de leite (em $\mathrm{kg}$ ) ao tempo $n$ (dias de lactação); $A$ é a produção de leite inicial $(\mathrm{kg}),{ }^{B}$ é a taxa de acréscimo de produção de leite $(\mathrm{kg})$ até o pico, $C$ é a taxa de declínio de produção $(\mathrm{kg})$ após o pico e exp é exponencial.

Para as análises via metodologia Bayesiana utilizou-se o software WinBUGS (SPIEGELHALTER, 1994), considerando-se prioris não informativas para os parâmetros. Foram geradas 30.000 amostras de cada parâmetro para cada raça, descartando-se os 3.000 primeiros, sendo as amostras retiradas a cada 30 iterações. As cadeias finais foram submetidas ao teste de convergência de Heidelberger; Welch (1983).

Entre as raças foram comparados os parâmetros da curva, a produção média diária e a produção total de leite no período avaliado. A partir dos parâmetros do modelo, também foram calculados o dia do pico $(P)$, e a produção de leite no pico $(P P)$; em que $P=B / C$ e $P P=A(B / C)^{B} * \exp (-B)$. 


\section{RESULTADOS E DISCUSSÃo}

A média dos dias produtivos das cabras Saanen foi de 205,78 dias, sendo o pico alcançado com 76,98 dias, com produção no pico de $3,48 \mathrm{~kg}$. Tais valores se assemelham aos encontrados por Zambom et al. (2005), que trabalhando com cabras leiteiras da raça Saanen, recebendo semelhante alimentação e mesma relação volumoso : concentrado deste trabalho, verificaram o pico aos 70,85 dias e uma produção de $3,42 \mathrm{~kg}$ no pico. Para os animais mestiços, a média de dias de produção foi de 147 , tendo o pico aos 17,08 dias e produção no pico de 2,04 kg de leite (Figura 1; Tabela 2).

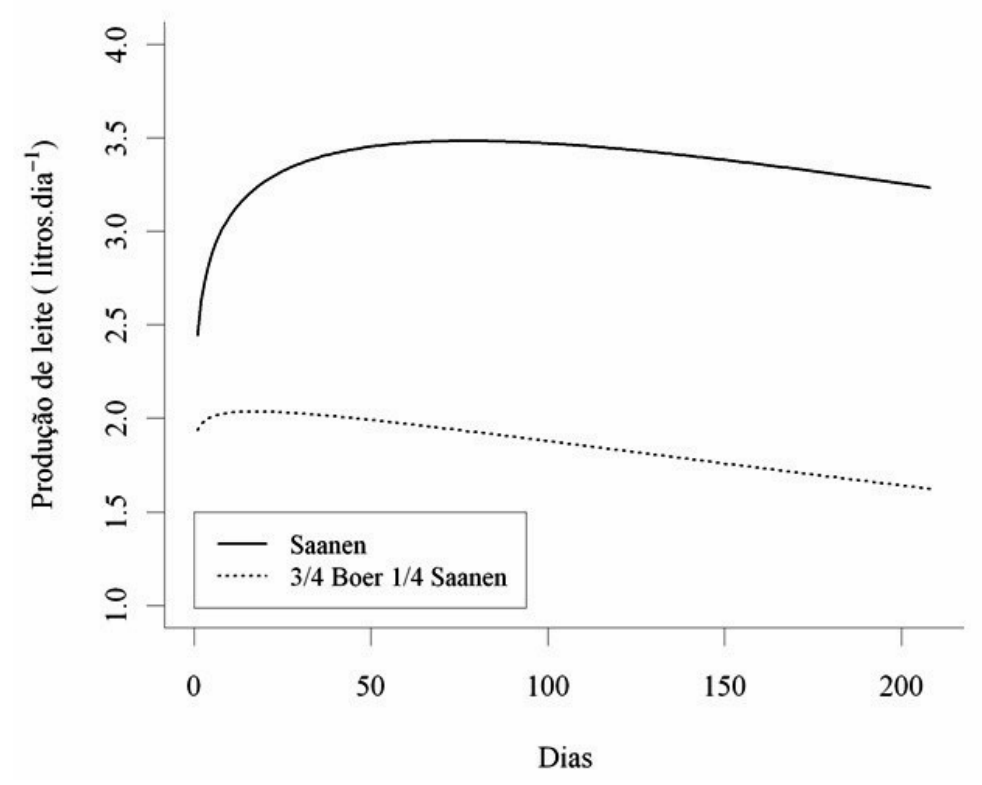

Figura 1. Curva de lactação de cabras Saanen e mestiças Boer-Saanen. 
Tabela 2. Produção no pico (PP) e tempo para atingir o pico (TP) de produção de cabras Saanen (S) e mestiças Boer-Saanen (B/S) estimados pelo modelo de Wood

\begin{tabular}{ccc}
\hline Raça & PP $(\mathrm{kg})$ & TP (dias) \\
\hline $\mathrm{S}$ & 3,48 & 76,98 \\
$\mathrm{~B} / \mathrm{S}$ & 2,04 & 17,08 \\
\hline
\end{tabular}

As produções totais e médias diárias foram calculadas com base nos parâmetros da curva, onde a produção foi ajustada para 208 dias, que é o número máximo de dias que as mestiças ficaram produzindo, e, então, foram comparadas para as duas raças. Os valores mostraram-se maiores, em ambas as comparações, para a raça Saanen, sendo eles, 699,6 kg e 3,363 kg, respectivamente, enquanto que para as cabras Boer-Saanen os valores foram $386,6 \mathrm{~kg}$ e $1,859 \mathrm{~kg}$, respectivamente. Os valores de produção média diária das cabras mestiças estão condizentes às informações de Casey; Niekerk (1988); Greyling et al. (2004), que citam que a produção de leite de cabras Boer varia de 1,5 a $2,5 \mathrm{~kg}$ por dia.

A produção total de leite das cabras Saanen encontrada foi maior que a relatada por Ribeiro (1997), que em estudo com animais desta raça criados na região de Andradas, Minas Gerais, encontrou valor de produção total média de $572,57 \mathrm{~kg}$ de leite.

Os resultados de produção se apresentam maiores que os encontrados por Soares Filho et al. (2001), que verificou em período de 219 dias de lactação, produção total de leite de 563,64 kg para animais da raça Saanen contra $319,97 \mathrm{~kg}$ para animais mestiços. Estes valores de produção dos animais mestiços, embora não se saiba quais as raças envolvidas no cruzamento, mostram-se mais próximos aos encontrados no presente trabalho.

$\mathrm{Na}$ determinação dos parâmetros da curva de lactação, verificou-se diferença significativa para os parâmetros $A$ e $B$, sendo eles, a produção inicial de leite $(\mathrm{kg})$ e a taxa de acréscimo $(\mathrm{kg})$ até o pico, respectivamente, maiores para os animais da raça Saanen. No entanto, para o parâmetro $C$ não se 
observou diferença, mostrando que a taxa de declínio da produção após o pico ocorreu de maneira semelhante para as duas raças. Isto mostra que os animais têm comportamento semelhante em relação à queda na produção, porém, o fato das cabras Saanen apresentarem uma produção inicial e uma taxa de acréscimo maior, faz com que sua produção no pico seja mais elevada e, mesmo que o valor de declínio seja semelhante ao das cabras mestiças, a raça Saanen apresenta maior produção (Tabela 3).

Tabela 3. Parâmetros da curva de lactação segundo modelo não linear de Wood e médias de produção.

\begin{tabular}{|c|c|c|c|c|c|}
\hline Parâmetro & $\operatorname{Raça}^{(1)}$ & Média & $\mathrm{Epm}^{(2)}$ & Icr $2,5 \%^{(3)}$ & $\operatorname{Icr} 97,5 \%^{(3)}$ \\
\hline \multirow{2}{*}{ A } & $\mathrm{S}$ & $2,4470{ }^{a}$ & 0,0728 & 2,3000 & 2,5845 \\
\hline & $B / S$ & $1,9405^{b}$ & 0,0933 & 1,74200 & 2,0940 \\
\hline \multirow{2}{*}{ B } & $S$ & $0,1055^{a}$ & 0,0098 & 0,0869 & 0,1258 \\
\hline & $B / S$ & $0,0261^{b}$ & 0,0166 & 0,0016 & 0,0625 \\
\hline \multirow{2}{*}{ C } & $\mathrm{S}$ & $0,0014^{a}$ & 0,0002 & 0,0011 & 0,0017 \\
\hline & $B / S$ & $0,0015 a$ & 0,0004 & 0,0009 & 0,0022 \\
\hline \multirow{2}{*}{ Produção Diária } & $S$ & $3,3630^{a}$ & 0,0106 & 3,3420 & 3,3840 \\
\hline & $B / S$ & $1,8590^{b}$ & 0,0093 & 1,8410 & 1,8770 \\
\hline \multirow{2}{*}{ Produção Total } & S & 699,600 a & 2,2030 & 695,200 & 703,800 \\
\hline & $B / S$ & $386,600^{b}$ & 1,9320 & 382,900 & 390,500 \\
\hline
\end{tabular}

A produção das cabras Saanen foi permanentemente maior em todos os momentos da curva, fazendo com que elas permaneçam por mais tempo na curva de lactação, concordando com Tekerli et al. (2000); Jakobsen et al. (2002); Stefanon et al. (2002), que afirmam que com maior persistência os 
animais produzem por mais tempo e por isso a persistência possui direta relação com aspectos econômicos da atividade leiteira. Desse modo, as cabras Saanen teriam maior interesse econômico em relação à produção de leite, uma vez que sua produção permanece maior que das mestiças, mesmo que o parâmetro $C$ tenha sido semelhante.

A média dos dias produtivos das duas raças mostra que as mestiças permaneceram por menos tempo em produção, uma vez que apresentaram menores valores para os parâmetros $A$ e $B$, além de alcançarem o pico mais rapidamente. Deste modo, a substituição de um rebanho leiteiro para um rebanho mestiço, pensando-se em utilizar os cabritos machos para abate e as fêmeas para produção leite, pode não ser a melhor solução, já que isso acarretaria em substituir uma grande produção de leite por uma média ou até mesmo baixa produção de leite e de carne. Esses resultados indicam que devese avaliar se o resultado econômico com o abate dos cabritos seria compensatório em relação à redução na produção leiteira.

\section{CONCLUSÕES}

Cabras mestiças Boer-Saanen apresentam diferente comportamento na curva de lactação, quando comparada às cabras Saanen puras, tendo menor produção inicial e menor taxa de acréscimo até o pico.

Cabras Saanen apresentaram maior produção em todas das fases da curva, consequentemente, maior persistência e maior produção de leite.

A abordagem Bayesiana permitiu estudar a da curva de lactação de cabras de maneira eficaz e estimar os parâmetros com acurácia.

\section{REFERÊNCIAS}

CARDOSO, L.L. Modelos de normas de reação para estudo da interação genótipo $x$ ambiente. 2009. 65p. Dissertação (Mestrado). Universidade Federal do Rio Grande do Sul. Porto Alegre, 2009. 
CASEY, N.H.; NIEKERK, W.A.V. The Boer goat. I. Origin, adaptability, performance, testing, reproduction and milk production. Small Ruminant Research, v.1, n.3, p.291-302, 1988.

CUNHA, D.N.F.V.; PEREIRA, J.C.; SILVA, F.F.; CAMPOS, O.F.; BRGA, J.L. MARTUSCELO. Selection of models of lactation curves to use in models of milk production simulation systems. Revista Brasileira de Zootecnia, v. 39, p. 891-202, 2010.

FARIA, C.U.; MAGNABOSCO, C.U.; REYES, A.L.; LÔBO, R.B.; BEZERRA, L.A.F. Inferência Bayesiana e sua aplicação na avaliação genética de bovinos da raça Nelore : revisão bibliográfica. Ciência Animal Brasileira, v. 8, n.1, p.75-86, 2007.

GLÓRIA， J.R.; BERGMANN, J.A.G.; QUIRINO, C.R.; RUAS, J.R.M.; MATOS, C.R.A.; PEREIRA, J.C.C. Curvas de lactação de quatro grupos genéticos de mestiças Holandês-Zebu. Revista Brasileira de Zootecnia, v.39, n.10, p.2160-2165, 2010.

GELMAN, A.; CARLIN, J.B.; STERN, H.S.; RUBIN, D.B. Bayesian data analysis. London: Chapman Hall, 1997. 526p.

GREYLING, J.P.C.; MMBENGWA, V.M.; SCWALBACH, L.M.J. Comparative Milk production potential of Indigenous and Boer goats under two feeding systems in South Africa. Small Ruminant Research, v.55, p.97-105, 2004.

GROENEWALD, P.C.N.; VILJOEN, C.S. A Bayesian model for analysis of lactation curves of dairy goats. Journal of Agricultural, Biological and Environmental Statistics, v.8, p.7583, 2003.

HEIDELBERGER, P.; WELCH, P.D. Simulation run length control in the presence of an initial transient. Operations Research, v.31, n.6, p.1109-1144, 1983.

JAKOBSEN, J.H.; MADSEN, P.; JENSEN, J. Genetic parameters for milk production and persistency for Danish Holstein estimated in random regression models using REML. Journal of Dairy Science, v.85, n.6, p.1607-1616, 2002.

MACEDO, V.P.; DAMASCENO, J.C.; SANTOS, G.T. Comportamento da curva de lactação de cabras mestiças Saanen em função da alocação de concentrado e do sistema de produção.

Revista Brasileira de Zootecnia, v.30, n.6, p.2093-2098, 2001.

MORAND-FEHR, P. Recent developments in goat nutrition and application: A review. Small Ruminant Research, v. 60, p. 25-43, 2005.

OLIVEIRA, H.T.V.; REIS, R.B.; GLÓRIA, J.R.; QUIRINO, C.R.; PEREIRA, J.C.C. Curvas de lactação de vacas F1 Holandês-Gir ajustadas pela função gama incompleta. Arquivo Brasileiro de Medicina Veterinária e Zootecnia, v. 59, p.233-238, 2007.

REKAYA, R.; CARABÃNO, M.J.; TORO, M.A. Bayesian analysis of lactation curves of HolsteinFriesian cattle using a nonlinear model. Journal of Dairy Science, v. 83, p. 2691-2701, 2000.

RIBEIRO, S. D. A. Caprinocultura: criação racional de caprinos. São Paulo: Nobel, 1997. $317 p$.

RIBEIRO, M.N.; PIMENTA FILHO, E.C. Estudo de efeitos ambientais que influem na forma da curva de lactação de cabras mestiças no estado da Paraíba. Revista Brasileira de Zootecnia, v.28, n.4, p.868-874, 1999. 
RODRIGUES, L.; SPINA, J.R., TEIXEIRA, I.A.M.A. Produção, composição do leite e exigências nutricionais de cabras Saanen em diferentes ordens de lactação. Acta Scientiarum, Animal Sciences, v.28, n.4, p.447-452, 2006.

SILVA, V.C. Composição do leite caprino e manejo correto na ordenha. Pubvet - Londrina, v.2, n.35, 2008.

SOARES FILHO, G.; MCMANUS, C.; MARIANTE, A.S. Fatores genéticos e ambientais que influenciam algumas características de reprodução e produção de leite de cabras no Distrito Federal. Revista Brasileira de Zootecnia, v.30, n.1, p.133-140, 2001.

SPIEGELHALTER, D. J. BUGS: Bayesian Inference using Gibbs Sampling. MRC Bioestatistics Unit, Cambridge, 1994.

STEFANON, B.; COLITTI, M.; GIANFRANCO, G. Mammary apoptosis and lactation persistency in dairy animals. Journal of Dairy Research, v.69, p.37-52, 2002.

VAL-ARREOLA, D.; KEBREAB, E.; DIJKSTRA, J.; FRANCE, J. Study of the lactation curve in dairy cattle on farms in Central Mexico. Journal of Dairy Science, v. 80, p. 3789-3799, 2004.

VANRADEN, P.M.; DEMATAWEWA, C.M.B.; PEARSON, R.E.; TOOKER, M.E. Productive life including all lactations and longer lactations with diminishing credits. Journal of Dairy Science, v.89, p. 3213-3220, 2006.

WOOD, P.D.P. Algebric model of lactation curve in cattle. Nature, v.216, n.511, p.164-165, 1967.

ZAMBOM, M.A.; ALCALDE, C.R.; MARTINS, E.N. Curva de lactação de cabras Saanen recebendo rações com diferentes relações volumoso:concentrado, 2002. Revista Brasileira de Zootecnia, v.34, n.6, p.2515-2521, 2005. 